\title{
REFINEMENTS OF SOME NUMERICAL RADIUS INEQUALITIES FOR HILBERT SPACE OPERATORS
}

\author{
MOHAMMAD .W. ALOMARI
}

\begin{abstract}
In this work, some generalizations and refinements inequalities for the numerical radius of the product of Hilbert space operators are proved. New inequalities for the numerical radius of block matrices of Hilbert space operators are also established.
\end{abstract}

\section{INTRODUCTION}

Let $\mathscr{B}(\mathscr{H})$ be the Banach algebra of all bounded linear operators defined on a complex Hilbert space $(\mathscr{H} ;\langle\cdot, \cdot\rangle)$ with the identity operator $1_{\mathscr{H}}$ in $\mathscr{B}(\mathscr{H})$.

For a bounded linear operator $T$ on a Hilbert space $\mathscr{H}$, the numerical range $W(T)$ is the image of the unit sphere of $\mathscr{H}$ under the quadratic form $x \rightarrow\langle T x, x\rangle$ associated with the operator. More precisely,

$$
W(T)=\{\langle T x, x\rangle: x \in \mathscr{H},\|x\|=1\} .
$$

Also, the numerical radius is defined to be

$$
w(T)=\sup \{|\lambda|: \lambda \in W(T)\}=\sup _{\|x\|=1}|\langle T x, x\rangle| .
$$

The spectral radius of an operator $T$ is defined to be

$$
r(T)=\sup \{|\lambda|: \lambda \in \operatorname{sp}(T)\} .
$$

We recall that, the usual operator norm of an operator $T$ is defined to be

$$
\|T\|=\sup \{\|T x\|: x \in H,\|x\|=1\}
$$

and

$$
\begin{aligned}
\ell(T): & =\inf \{\|T x\|: x \in \mathscr{H},\|x\|=1\} \\
& =\inf \{|\langle T x, y\rangle|: x, y \in \mathscr{H},\|x\|=\|y\|=1\} .
\end{aligned}
$$

Date: March 18, 2019.

2010 Mathematics Subject Classification. Primary: 47A12, 47A30 Secondary: 15A60, 47A63.

Key words and phrases. Numerical radius, Operator norm, mixed Schwarz inequality. 
It's well known that the numerical radius is not submultiplicative, but it is satisfies $w(T S) \leq 4 w(T) w(S)$ for all $T, S \in \mathscr{B}(\mathscr{H})$. In particular if $T, S$ are commute, then $w(T S) \leq 2 w(T) w(S)$. Moreover, if $T, S$ are normal then $w(\cdot)$ is submultiplicative $w(T S) \leq w(T) w(S)$. Denote $|T|=\left(T^{*} T\right)^{1 / 2}$ the absolute value of the operator $T$. Then we have $w(|T|)=\|T\|$. It's convenient to mention that, the numerical radius norm is weakly unitarily invariant; i.e., $w\left(U^{*} T U\right)=w(T)$ for all unitary $U$. Also, let us not miss the chance to mention the important property that $w(T)=w\left(T^{*}\right)$ and $w\left(T^{*} T\right)=w\left(T T^{*}\right)$ for every $T \in \mathscr{B}(\mathscr{H})$.

A popular problem is the following: does the numerical radius of the product of operators commute, i.e., $w(T S)=w(S T)$ for any operators $T, S \in \mathscr{B}(\mathscr{H})$ ?

This problem has been given serious attention by many authors and in several resources (see [14], for example). Fortunately, it has been shown recently that, for one of such operators must be a multiple of a unitary operator, and we need only to check $w(T S)=w(S T)$ for all rank one operators $S \in \mathscr{B}(\mathscr{H})$ to arrive at the conclusion. This fact was proved by Chien et al. in [6]. For other related problems involving numerical ranges and radius see [6] and [7] as well as the elegant work of $\mathrm{Li}$ [27] and the references therein. For more classical and recent properties of numerical range and radius, see [6] [7], [27] and the comprehensive books [4], [16] and [17].

On the other hand, it is well known that $w(\cdot)$ defines an operator norm on $\mathscr{B}(\mathscr{H})$ which is equivalent to operator norm $\|\cdot\|$. Moreover, we have

$$
\frac{1}{2}\|T\| \leq w(T) \leq\|T\|
$$

for any $T \in \mathscr{B}(\mathscr{H})$. The inequality is sharp.

In 2003, Kittaneh [21] refined the right-hand side of (1.1), where he proved that

$$
w(T) \leq \frac{1}{2}\left(\|T\|+\left\|T^{2}\right\|^{1 / 2}\right)
$$

for any $T \in \mathscr{B}(\mathscr{H})$.

After that in 2005, the same author in [19] proved that

$$
\frac{1}{4}\left\|A^{*} A+A A^{*}\right\| \leq w^{2}(A) \leq \frac{1}{2}\left\|A^{*} A+A A^{*}\right\| .
$$

The inequality is sharp. This inequality was also reformulated and generalized in [13] but in terms of Cartesian decomposition. 
In 2007, Yamazaki [30] improved (1.1) by proving that

$$
w(T) \leq \frac{1}{2}(\|T\|+w(\widetilde{T})) \leq \frac{1}{2}\left(\|T\|+\left\|T^{2}\right\|^{1 / 2}\right)
$$

where $\widetilde{T}=|T|^{1 / 2} U|T|^{1 / 2}$ with unitary $U$.

In 2008, Dragomir [11] used Buzano inequality to improve (1.1), where he proved that

$$
w^{2}(T) \leq \frac{1}{2}\left(\|T\|+w\left(T^{2}\right)\right) .
$$

This result was also recently generalized by Sattari et al. in [29].

This work, is divided into four sections, after this introduction, in Section 2, we recall some well-known inequalities for bounded linear operators. In Section 3, some generalizations and refinements of the numerical radius inequalities are proved. In Section 4, new refinement inequalities for the numerical radius of $n \times n$ Hilbert space operator matrices are established.

\section{LEMMAS}

In order to prove our results we need a sequence of lemmas.

Lemma 1. We have

(1) The Power-Mean inequality

$$
a^{\alpha} b^{1-\alpha} \leq \alpha a+(1-\alpha) b \leq\left(\alpha a^{p}+(1-\alpha) b^{p}\right)^{\frac{1}{p}},
$$

for all $\alpha \in[0,1], a, b \geq 0$ and $p \geq 1$.

(2) The Power-Young inequality

$$
a b \leq \frac{a^{\alpha}}{\alpha}+\frac{b^{\beta}}{\beta} \leq\left(\frac{a^{p \alpha}}{\alpha}+\frac{b^{p \beta}}{\beta}\right)^{\frac{1}{p}}
$$

for all $a, b \geq 0$ and $\alpha, \beta>1$ with $\frac{1}{\alpha}+\frac{1}{\beta}=1$ and all $p \geq 1$.

Lemma 2. (The McCarty inequality). Let $A \in \mathscr{B}(\mathscr{H})^{+}$, then

$$
\langle A x, x\rangle^{p} \leq\left\langle A^{p} x, x\right\rangle, \quad p \geq 1
$$

for any unit vector $x \in \mathscr{H}$

The mixed Schwarz inequality was introduced in [25], as follows:

Lemma 3. Let $A \in \mathscr{B}(\mathscr{H})^{+}$, then

$$
|\langle A x, y\rangle|^{2} \leq\left\langle|A|^{2 \alpha} x, x\right\rangle\left\langle\left|A^{*}\right|^{2(1-\alpha)} y, y\right\rangle, \quad 0 \leq \alpha \leq 1 .
$$

for any vectors $x, y \in \mathscr{H}$ 
In order to generalize (2.4), Kittaneh in [24] used the following key lemma to prove a generalization of Kato's inequality (2.4).

Lemma 4. Let $A, B \in \mathscr{B}(\mathscr{H})^{+}$. Then $\left[\begin{array}{cc}A & C^{*} \\ C & B\end{array}\right]$ is positive in $\mathscr{B}(\mathscr{H} \oplus \mathscr{H})$ if and only if $|\langle C x, y\rangle|^{2} \leq\langle A x, x\rangle\langle B y, y\rangle$ for every vectors $x, y \in \mathscr{H}$.

Indeed, in [24] we find that

Lemma 5. Let $A, B \in \mathscr{B}(\mathscr{H})$ such that $|A| B=B^{*}|A|$. If $f$ and $g$ are nonnegative continuous functions on $[0, \infty)$ satisfying $f(t) g(t)=t$ $(t \geq 0)$, then

$$
|\langle A B x, y\rangle| \leq r(B)\|f(|A|) x\|\left\|g\left(\left|A^{*}\right|\right) y\right\|
$$

for any vectors $x, y \in \mathscr{H}$.

Clearly, by setting $B=1_{\mathscr{H}}$ and choosing $f(t)=t^{\alpha}, g(t)=t^{1-\alpha}$, then the inequality (2.5) reduces to (2.4).

The following useful estimate of a spectral radius was also obtained by Kittaneh in [20].

Lemma 6. If $A, B \in \mathscr{B}(\mathscr{H})$. Then

$$
\leq \frac{1}{4}\left(\|A B\|+\|B A\|+\sqrt{(\|A B\|-\|B A\|)^{2}+4 m(A, B)}\right),
$$

where $m(A, B):=\min \{\|A\|\|B A B\|,\|B\|\|A B A\|\}$.

In some of our results we need the following two fundamental norm estimates, which are:

$$
\begin{aligned}
& \|A+B\| \\
& \quad \leq \frac{1}{2}\left(\|A\|+\|B\|+\sqrt{(\|A\|-\|B\|)^{2}+4\left\|A^{1 / 2} B^{1 / 2}\right\|^{2}}\right),
\end{aligned}
$$

and

$$
\left\|A^{1 / 2} B^{1 / 2}\right\| \leq\|A B\|^{1 / 2}
$$

Both estimates are valid for all positive operators $A, B \in \mathscr{B}(\mathscr{H})$. Also, it should be noted that (2.7) is sharper than the triangle inequality as pointed out by Kittaneh in [23].

A new refinement of Cauchy-Schwarz inequality was recently obtained in [3], as follows: 
NUMERICAL RADIUS INEQUALITIES FOR HILBERT SPACE OPERATORS 5

Lemma 7. Let $A \in \mathscr{B}(\mathscr{H})^{+}$, then

$$
\begin{aligned}
|\langle A x, y\rangle|^{2 p} \leq & {\left[\left\langle A^{p} x, x\right\rangle-\left\langle\left|A-\langle A x, x\rangle 1_{\mathcal{H}}\right|^{p} x, x\right\rangle\right] } \\
& \times\left[\left\langle A^{p} y, y\right\rangle-\left\langle\left|A-\langle A y, y\rangle 1_{\mathcal{H}}\right|^{p} y, y\right\rangle\right] \\
\leq & \left\langle A^{p} x, x\right\rangle\left\langle A^{p} y, y\right\rangle
\end{aligned}
$$

for all $p \geq 2$ and every $x, y \in \mathcal{H}$.

\section{Numerical Radius Inequalities}

Let us begin with the following result.

Theorem 1. Let $A, B \in \mathscr{B}(\mathscr{H})$ such that $|A| B=B^{*}|A|$. If $f, g$ be nonnegative continuous functions on $[0, \infty)$ satisfying $f(t) g(t)=t$, $(t \geq 0)$. Then

$$
\begin{aligned}
w(A B) \leq & \frac{1}{2} r(B) w\left(\left(f^{2}(|A|)+g^{2}\left(\left|A^{*}\right|\right)\right)\right) \\
\leq & \frac{1}{8}\left(\|B\|+\left\|B^{2}\right\|^{1 / 2}\right)\left\{\left\|f^{2}(|A|)\right\|+\left\|g^{2}\left(\left|A^{*}\right|\right)\right\|\right. \\
& \left.\quad+\sqrt{\left(\left\|f^{2}(|A|)\right\|-\left\|g^{2}\left(\left|A^{*}\right|\right)\right\|\right)^{2}+4\left\|f(|A|) g\left(\left|A^{*}\right|\right)\right\|^{2}}\right\} .
\end{aligned}
$$

In particular, we have

$$
w(A) \leq \frac{1}{2} w\left(\left(f^{2}(|A|)+g^{2}\left(\left|A^{*}\right|\right)\right)\right) .
$$

Proof. Setting $y=x$ in (2.5), we get

$$
\begin{aligned}
|\langle A B x, x\rangle| & \leq r(B)\|f(|A|) x\|\left\|g\left(\left|A^{*}\right|\right) x\right\| \\
& =r(B)\left\langle f^{2}(|A|) x, x\right\rangle^{1 / 2}\left\langle g^{2}\left(\left|A^{*}\right|\right) x, x\right\rangle^{1 / 2} \\
& \leq \frac{1}{2} r(B)\left(\left\langle f^{2}(|A|) x, x\right\rangle+\left\langle g^{2}\left(\left|A^{*}\right|\right) x, x\right\rangle\right) \quad \text { by }(2.1) \\
& =\frac{1}{2} r(B)\left\langle\left(f^{2}(|A|)+g^{2}\left(\left|A^{*}\right|\right)\right) x, x\right\rangle
\end{aligned}
$$

Thus, by taking the supremum over $x \in \mathscr{H}$ we get that

$$
\begin{aligned}
\sup _{\|x\|=1}|\langle A B x, x\rangle| & \leq \frac{1}{2} r(B) \sup _{\|x\|=1}\left\langle\left(f^{2}(|A|)+g^{2}\left(\left|A^{*}\right|\right)\right) x, x\right\rangle \\
& =\frac{1}{2} r(B) w\left(\left(f^{2}(|A|)+g^{2}\left(\left|A^{*}\right|\right)\right)\right) \\
& \left(\leq \frac{1}{2} r(B)\left\|\left(f^{2}(|A|)+g^{2}\left(\left|A^{*}\right|\right)\right)\right\|\right)
\end{aligned}
$$


which proves the first inequality in (3.1).

$$
\begin{aligned}
\sup _{\|x\|=1}|\langle A B x, x\rangle| & \leq \frac{1}{2} r(B) w\left(\left(f^{2}(|A|)+g^{2}\left(\left|A^{*}\right|\right)\right)\right) \\
& \leq \frac{1}{2} r(B)\left\|\left(f^{2}(|A|)+g^{2}\left(\left|A^{*}\right|\right)\right)\right\|
\end{aligned}
$$

The second inequality follows by employing the (2.7) on the last inequality above i.e.,

$$
\begin{aligned}
\left\|\left(f^{2}(|A|)+g^{2}\left(\left|A^{*}\right|\right)\right)\right\| \leq & \frac{1}{2} r(B)\left\{\left\|f^{2}(|A|)\right\|+\left\|g^{2}\left(\left|A^{*}\right|\right)\right\|\right. \\
& \left.+\sqrt{\left(\left\|f^{2}(|A|)\right\|-\left\|g^{2}\left(\left|A^{*}\right|\right)\right\|\right)^{2}+4\left\|f(|A|) g\left(\left|A^{*}\right|\right)\right\|^{2}}\right\}
\end{aligned}
$$

also, by using (2.6) with $A=1_{\mathscr{H}}$, we get

$$
\begin{aligned}
\sup _{\|x\|=1}|\langle A B x, x\rangle| \leq \frac{1}{8} & \left(\|B\|+\left\|B^{2}\right\|^{1 / 2}\right)\left\{\left\|f^{2}(|A|)\right\|+\left\|g^{2}\left(\left|A^{*}\right|\right)\right\|\right. \\
& \left.+\sqrt{\left(\left\|f^{2}(|A|)\right\|-\left\|g^{2}\left(\left|A^{*}\right|\right)\right\|\right)^{2}+4\left\|f(|A|) g\left(\left|A^{*}\right|\right)\right\|^{2}}\right\}
\end{aligned}
$$

and this proves the second inequality in (3.1).

Corollary 1. Let $A, B \in \mathscr{B}(\mathscr{H})$ such that $|A| B=B^{*}|A|$. Then,

$$
\begin{aligned}
w(A B) \leq & \frac{1}{2} r(B) w\left(\left(|A|^{2 \alpha}+\left|A^{*}\right|^{2(1-\alpha)}\right)\right) \\
\leq & \frac{1}{8}\left(\|B\|+\left\|B^{2}\right\|^{1 / 2}\right)\left\{\left\||A|^{2 \alpha}\right\|+\left\|\left|A^{*}\right|^{2(1-\alpha)}\right\|\right. \\
& \left.+\sqrt{\left(\left\||A|^{2 \alpha}\right\|-\left\|\left|A^{*}\right|^{2(1-\alpha)}\right\|\right)^{2}+4\left\||A|^{\alpha}\left|A^{*}\right|^{1-\alpha}\right\|^{2}}\right\} .
\end{aligned}
$$

for all $0 \leq \alpha \leq 1$.

Proof. Setting $f(t)=t^{\alpha}$ and $g(t)=t^{1-\alpha}, 0 \leq \alpha \leq 1, t \geq 0$ in Theorem 1.

Corollary 2. Let $A, B \in \mathscr{B}(\mathscr{H})$ such that $|A| B=B^{*}|A|$. In particular, we have

$$
w(A B) \leq \frac{1}{4}\left(\|B\|+\left\|B^{2}\right\|^{1 / 2}\right) \cdot\left(\|A\|+\left\|A^{2}\right\|^{1 / 2}\right)
$$


Proof. Setting $\alpha=\frac{1}{2}$ in (3.2) we get

$$
\begin{aligned}
w(A B) \leq & \frac{1}{2} r(B) w\left(\left(|A|^{2 \alpha}+\left|A^{*}\right|^{2(1-\alpha)}\right)\right) \\
\leq & \frac{1}{8}\left(\|B\|+\left\|B^{2}\right\|^{1 / 2}\right)\left\{\||A|\|+\left\|\left|A^{*}\right|\right\| \quad\left(\text { by }(2.6) \text { with } A=1_{\mathscr{H}}\right)\right. \\
& \left.\quad+\sqrt{\left(\||A|\|-\left\|\left|A^{*}\right|\right\|\right)^{2}+4\left\||A|^{1 / 2}\left|A^{*}\right|^{1 / 2}\right\|^{2}}\right\} . \\
= & \frac{1}{4}\left(\|B\|+\left\|B^{2}\right\|^{1 / 2}\right) \cdot\left(\|A\|+\left\|A^{2}\right\|^{1 / 2}\right)
\end{aligned}
$$

where the last inequality follows from (2.8) and using the fact that $\||A|\|=\left\|A^{*} \mid\right\|=\|A\|$ and this proves the desired result.

A generalization of Theorem 1 is given as follows:

Theorem 2. Let $A, B \in \mathscr{B}(\mathscr{H})$ such that $|A| B=B^{*}|A|$. If $f, g$ be nonnegative continuous functions on $[0, \infty)$ satisfying $f(t) g(t)=t$, $(t \geq 0)$. Then,

$$
\begin{aligned}
w^{p}(A B) & \leq r^{p}(B) \cdot w\left(\frac{1}{\alpha} f^{\alpha p}(|A|)+\frac{1}{\beta} g^{\beta p}\left(\left|A^{*}\right|\right)\right) \\
& \leq r^{p}(B) \cdot\left\|\frac{1}{\alpha} f^{\alpha p}(|A|)+\frac{1}{\beta} g^{\beta p}\left(\left|A^{*}\right|\right)\right\|
\end{aligned}
$$

for all $p \geq 1, \alpha \geq \beta>1$ with $\frac{1}{\alpha}+\frac{1}{\beta}=1$ and $\beta p \geq 2$. Moreover we have

$$
\begin{aligned}
w^{p}(A B) & \leq \frac{1}{2^{p+1}} \cdot \gamma \cdot\left(\|B\|+\left\|B^{2}\right\|^{1 / 2}\right)^{p} \\
& \times\left\{\left\|f^{\alpha p}(|A|)\right\|+\left\|g^{\beta p}\left(\left|A^{*}\right|\right)\right\|+\Phi(f, g ; A)\right\},
\end{aligned}
$$

where $\Phi(f, g ; A):=\sqrt{\left[\left|f^{\alpha p}(|A|)\right|-\left\|g^{\beta p}\left(\left|A^{*}\right|\right)\right\|\right]^{2}+4\left\|f^{p \alpha}(|A|) g^{p \beta}\left(\left|A^{*}\right|\right)\right\|}$ and $\gamma=\max \left\{\frac{1}{\alpha}, \frac{1}{\beta}\right\}$. 
Proof. Using the mixed Schwarz inequality (2.5), we have

$$
\begin{aligned}
& |\langle A B x, x\rangle|^{p} \\
& \leq r^{p}(B)\|f(|A|) x\|^{p}\left\|g\left(\left|A^{*}\right|\right) x\right\|^{p} \\
& =r^{p}(B)\left\langle f^{2}(|A|) x, x\right\rangle^{\frac{p}{2}}\left\langle g^{2}\left(\left|A^{*}\right|\right) x, x\right\rangle^{\frac{p}{2}} \\
& \leq r^{p}(B)\left[\frac{1}{\alpha}\left\langle f^{2}(|A|) x, x\right\rangle^{\frac{\alpha p}{2}}+\frac{1}{\beta}\left\langle g^{2}\left(\left|A^{*}\right|\right) x, x\right\rangle^{\frac{\beta p}{2}}\right] \\
& \leq r^{p}(B)\left[\frac{1}{\alpha}\left\langle f^{\alpha p}(|A|) x, x\right\rangle+\frac{1}{\beta}\left\langle g^{\beta p}\left(\left|A^{*}\right|\right) x, x\right\rangle\right] \\
& =r^{p}(B)\left\langle\left[\frac{1}{\alpha} f^{\alpha p}(|A|)+\frac{1}{\beta} g^{\beta p}\left(\left|A^{*}\right|\right)\right] x, x\right\rangle .
\end{aligned}
$$

Taking the supremum over $x \in \mathscr{H}$, we obtain the first inequality in (3.4). To obtain the second inequality, by utilizing (1.1) on the first inequality in (3.4) we have

$$
\begin{aligned}
& w\left(\frac{1}{\alpha} f^{\alpha p}(|A|)+\frac{1}{\beta} g^{\beta p}\left(\left|A^{*}\right|\right)\right) \\
& \leq\left\|\frac{1}{\alpha} f^{\alpha p}(|A|)+\frac{1}{\beta} g^{\beta p}\left(\left|A^{*}\right|\right)\right\| \\
& \leq \max \left\{\frac{1}{\alpha}, \frac{1}{\beta}\right\} \cdot\left\|f^{\alpha p}(|A|)+g^{\beta p}\left(\left|A^{*}\right|\right)\right\| \\
& \leq \frac{1}{2} \gamma\left(\left\|f^{\alpha p}(|A|)\right\|+\left\|g^{\beta p}\left(\left|A^{*}\right|\right)\right\| \quad \quad \text { by } \quad(2.7)\right) \\
& \left.\quad+\sqrt{\left[\left|f^{\alpha p}(|A|)\right|-\left\|g^{\beta p}\left(\left|A^{*}\right|\right)\right\|\right]^{2}+4\left\|f^{p \alpha / 2}(|A|) g^{p \beta / 2}\left(\left|A^{*}\right|\right)\right\|^{2}}\right) \\
& \leq \frac{1}{2} \gamma\left(\left\|f^{\alpha p}(|A|)\right\|+\left\|g^{\beta p}\left(\left|A^{*}\right|\right)\right\|\right. \\
& \left.\quad+\sqrt{\left[\left|f^{\alpha p}(|A|)\right|-\left\|g^{\beta p}\left(\left|A^{*}\right|\right)\right\|\right]^{2}+4\left\|f^{p \alpha}(|A|) g^{p \beta}\left(\left|A^{*}\right|\right)\right\|}\right)
\end{aligned}
$$

Hence, by substituting all in (3.4) we get

$$
\begin{aligned}
w^{p}(A B) \leq & r^{p}(B) \cdot w\left(\frac{1}{\alpha} f^{\alpha p}(|A|)+\frac{1}{\beta} g^{\beta p}\left(\left|A^{*}\right|\right)\right) \\
\leq & r^{p}(B) \cdot\left\|\frac{1}{\alpha} f^{\alpha p}(|A|)+\frac{1}{\beta} g^{\beta p}\left(\left|A^{*}\right|\right)\right\| \\
\leq & \frac{1}{2^{p+1}} \cdot \gamma \cdot\left(\|B\|+\left\|B^{2}\right\|^{1 / 2}\right)^{p} \\
& \quad \times\left\{\left\|f^{\alpha p}(|A|)\right\|+\left\|g^{\beta p}\left(\left|A^{*}\right|\right)\right\|+\Phi(f, g ; A)\right\},
\end{aligned}
$$


where the last inequality follows from (2.6) with $A=1_{\mathscr{H}}$ and this is yield the required result, where $\Phi(f, g ; A)$ is defined above.

A generalization of Sattari et al.inequality which was obtained in [29] is given as follows:

Theorem 3. Let $A, B \in \mathscr{B}(\mathscr{H})$ such that

(1) $A B=B A$, and

(2) $\left|A^{2}\right| B^{2}=\left(B^{2}\right)^{*}\left|A^{2}\right|$.

If $f, g$ be nonnegative continuous functions on $[0, \infty)$ satisfying $f(t) g(t)=$ $t,(t \geq 0)$. Then,

$$
\begin{aligned}
w^{2 p}(A B) \leq \frac{1}{2}\|A B\|^{2 p}+\frac{\gamma}{2^{p+2}}\left(\left\|B^{2}\right\|+\left\|B^{4}\right\|^{1 / 2}\right)^{p} \\
\times\left\{\left\|f^{\alpha p}(|A|)\right\|+\left\|g^{\beta p}\left(\left|A^{*}\right|\right)\right\|+\Phi(f, g ; A)\right\}
\end{aligned}
$$

where $\Phi(f, g ; A)$ is defined in Theorem 2, and $\gamma=\max \left\{\frac{1}{\alpha}, \frac{1}{\beta}\right\}$, for all $p \geq 1, \alpha \geq \beta>1$ with $\frac{1}{\alpha}+\frac{1}{\beta}=1$ and $\beta p \geq 2$.

Proof. Let us first note that the Dragomir refinement of Cauchy-Schwarz inequality reads that [12]:

$$
|\langle x, y\rangle| \leq|\langle x, e\rangle\langle e, y\rangle|+|\langle x, y\rangle-\langle x, e\rangle\langle e, y\rangle| \leq\|x\|\|y\|
$$

for all $x, y, e \in \mathscr{H}$ with $\|e\|=1$.

It's easy to deduce the inequality

$$
|\langle x, e\rangle\langle e, y\rangle| \leq \frac{1}{2}(|\langle x, y\rangle|+\|x\|\|y\|) .
$$

Setting $e=u, x=A B u, y=B^{*} A^{*} u$ in (3.7) and using the Power-Mean inequality (2.1) we get

$$
\begin{aligned}
\left|\langle A B u, u\rangle\left\langle u, B^{*} A^{*} u\right\rangle\right| & \leq \frac{1}{2}\left(\left|\left\langle A B u, B^{*} A^{*} u\right\rangle\right|+\|A u\|\left\|B^{*} A^{*} u\right\|\right) \\
& \leq\left(\frac{\left|\left\langle(A B)^{2} u, u\right\rangle\right|^{p}+\|A u\|^{p}\left\|A^{*} u\right\|^{p}}{2^{p}}\right)^{\frac{1}{p}}
\end{aligned}
$$

since $A, B$ are commutative so that $(A B)^{2}=A^{2} B^{2}$. Equivalently, we may write

$$
|\langle A B u, u\rangle|^{2 p} \leq \frac{1}{2}\left(\left|\left\langle(A B)^{2} u, u\right\rangle\right|^{p}+\|A B u\|^{p}\left\|B^{*} A^{*} u\right\|^{p}\right) .
$$


Now, using the mixed Schwarz inequality (2.5) by replacing $A, B$ by $A^{2}, B^{2}$; respectively, then we have

$$
\begin{aligned}
& \left|\left\langle(A B)^{2} x, x\right\rangle\right|^{p} \\
& \leq r^{p}\left(B^{2}\right)\left\|f\left(\left|A^{2}\right|\right) x\right\|^{p}\left\|g\left(\left|\left(A^{2}\right)^{*}\right|\right) x\right\|^{p} \\
& =r^{p}\left(B^{2}\right)\left\langle f^{2}\left(\left|A^{2}\right|\right) x, x\right\rangle^{\frac{p}{2}}\left\langle g^{2}\left(\left|\left(A^{2}\right)^{*}\right|\right) x, x\right\rangle^{\frac{p}{2}} \\
& \leq r^{p}\left(B^{2}\right)\left[\frac{1}{\alpha}\left\langle f^{2}\left(\left|A^{2}\right|\right) x, x\right\rangle^{\frac{\alpha p}{2}}+\frac{1}{\beta}\left\langle g^{2}\left(\left|\left(A^{2}\right)^{*}\right|\right) x, x\right\rangle^{\frac{\beta p}{2}}\right] \\
& \leq r^{p}\left(B^{2}\right)\left[\frac{1}{\alpha}\left\langle f^{\alpha p}\left(\left|A^{2}\right|\right) x, x\right\rangle+\frac{1}{\beta}\left\langle g^{\beta p}\left(\left|\left(A^{2}\right)^{*}\right|\right) x, x\right\rangle\right] \\
& =r^{p}\left(B^{2}\right)\left\langle\left[\frac{1}{\alpha} f^{\alpha p}\left(\left|A^{2}\right|\right)+\frac{1}{\beta} g^{\beta p}\left(\left|\left(A^{2}\right)^{*}\right|\right)\right] x, x .\right\rangle .
\end{aligned}
$$

Substituting in (3.8) and taking the supremum over $x \in \mathscr{H}$, and finally proceed as in the proof of Theorem 2 we get the desired inequality. We shall omit the details.

Corollary 3. Under the assumptions of Theorem 3, we have

$$
w^{2}(A B) \leq \frac{1}{2}\|A B\|^{2}+\frac{1}{8}\left(\left\|B^{2}\right\|+\left\|B^{4}\right\|^{1 / 2}\right)\left(\|A\|+\left\|A^{2}\right\|^{1 / 2}\right)
$$

Theorem 4. Let $A, B \in \mathscr{B}(\mathscr{H})^{+}$such that $A B$ is contraction. Then

$$
\begin{aligned}
w^{2 p}(A B) \leq\left[\left\|A^{p}\right\|-\ell\left(\left\||(A-\|A\|)|^{p}\right\|\right)\right] & \\
& \times\left[\left\|B^{p}\right\|-\ell\left(\left\||(B-\|B\|)|^{p}\right\|\right)\right]
\end{aligned}
$$

for all $p \geq 2$. In particular, we have

$$
w^{2 p}(A) \leq\left\|A^{p}\right\|-\ell\left(\left\||(A-\|A\|)|^{p}\right\|\right)
$$

for every positive contraction $A$.

Proof. Let us first prove that $T=\left[\begin{array}{cc}A & B^{*} A^{*} \\ A B & B\end{array}\right] \in \mathscr{B}(\mathscr{H} \oplus \mathscr{H})$ is positive. Since $A B$ is contraction, then by Proposition I.3.5 ([4], p. 10), $\left[\begin{array}{cc}I & B^{*} A^{*} \\ A B & I\end{array}\right]$ is positive. Thus,

$$
T=\left[\begin{array}{cc}
A & B^{*} A^{*} \\
A B & B
\end{array}\right]=\left[\begin{array}{cc}
A & 0 \\
0 & B
\end{array}\right]+\left[\begin{array}{cc}
0 & B^{*} A^{*} \\
A B & 0
\end{array}\right]
$$

is positive since $A, B \geq 0$. 
Now, let $\mathbf{x}=\left(\begin{array}{l}x_{1} \\ x_{2}\end{array}\right)$ in $\mathscr{H} \oplus \mathscr{H}$, such that $\left\|x_{1}\right\|^{2}+\left\|x_{2}\right\|^{2}=1$. Since $A B$ is contraction then $\left[\begin{array}{cc}A & B^{*} A^{*} \\ A B & B\end{array}\right]$ is positive. Therefore, by setting $C=A B$ in Lemma 4, and this implies that

$$
\left|\left\langle A B x_{1}, x_{2}\right\rangle\right|^{2 p} \leq\left\langle A x_{1}, x_{1}\right\rangle^{p}\left\langle B x_{2}, x_{2}\right\rangle^{p}
$$

If we wish setting $x_{1}=x_{2}$ and employing the first inequality in Lemma 7, we get

$$
\begin{aligned}
\left|\left\langle A B x_{1}, x_{2}\right\rangle\right|^{2 p} \leq & \left\langle A x_{1}, x_{1}\right\rangle^{p}\left\langle B x_{2}, x_{2}\right\rangle^{p} \\
\leq & \left(\left\langle A^{p} x_{1}, x_{1}\right\rangle-\left\langle\left|A-\left\langle A x_{1}, x_{1}\right\rangle 1_{\mathcal{H}}\right|^{p} x_{1}, x_{1}\right\rangle\right) \\
& \quad \times\left(\left\langle B^{p} x_{2}, x_{2}\right\rangle-\left\langle\left|B-\left\langle B x_{2}, x_{2}\right\rangle 1_{\mathcal{H}}\right|^{p} x_{2}, x_{2}\right\rangle\right) \\
\leq & \left(\left\langle A^{p} x_{1}, x_{1}\right\rangle-\left\langle\left|A-\sup _{\left\|x_{1}\right\|=1}\left\langle A x_{1}, x_{1}\right\rangle 1_{\mathcal{H}}\right|^{p} x_{1}, x_{1}\right\rangle\right) \\
& \times\left(\left\langle B^{p} x_{2}, x_{2}\right\rangle-\left\langle\left|B-\sup _{\left\|x_{2}\right\|=1}\left\langle B x_{2}, x_{2}\right\rangle 1_{\mathcal{H}}\right|^{p} x_{2}, x_{2}\right\rangle\right)
\end{aligned}
$$

Taking the supremum over $x_{1}, x_{2} \in \mathscr{H}$, we observe that

$$
\begin{aligned}
& \sup _{\left\|x_{1}\right\|=\left\|x_{2}\right\|=1}\left|\left\langle A B x_{1}, x_{2}\right\rangle\right|^{2 p} \\
& \leq \sup _{\left\|x_{1}\right\|=\left\|x_{2}\right\|=1}\left\{\left[\left\langle A^{p} x_{1}, x_{1}\right\rangle-\left\langle\left|A-\sup _{\left\|x_{1}\right\|=1}\left\langle A x_{1}, x_{1}\right\rangle 1_{\mathcal{H}}\right|^{p} x_{1}, x_{1}\right\rangle\right]\right. \\
& \left.\quad \times\left[\left\langle B^{p} x_{2}, x_{2}\right\rangle-\left\langle\left|B-\sup _{\left\|x_{2}\right\|=1}\left\langle B x_{2}, x_{2}\right\rangle 1_{\mathcal{H}}\right| x_{2}, x_{2}\right\rangle\right]\right\} \\
& \leq \sup _{\left\|x_{1}\right\|=1}\left\langle A^{p} x_{1}, x_{1}\right\rangle-\inf _{\left\|x_{1}\right\|=1}\left\langle\left|A-\sup _{\left\|x_{1}\right\|=1}\left\langle A x_{1}, x_{1}\right\rangle 1_{\mathcal{H}}\right|^{\left.x_{1}, x_{1}\right\rangle}\right\rangle \\
& \quad \times \sup _{\left\|x_{1}\right\|=1}\left\langle B^{p} x_{2}, x_{2}\right\rangle-\inf _{\left\|x_{2}\right\|=1}\left\langle\left|B-\sup _{\left\|x_{2}\right\|=1}\left\langle B x_{2}, x_{2}\right\rangle 1_{\mathcal{H}}\right|_{x_{2}, x_{2}}\right\rangle \\
& =\left[\left\|A^{p}\right\|-\ell\left(\left.\|\|(A-\|A\|)\right|^{p} \|\right)\right] \times\left[\left\|B^{p}\right\|-\ell\left(\left\||(B-\|B\|)|^{p}\right\|\right)\right]
\end{aligned}
$$

which completes the proof.

As we have seen, the positivity assumption of $T=\left[\begin{array}{cc}A & B^{*} A^{*} \\ A B & B\end{array}\right]$ in Theorem 4 is essential. A more general case could be obtained for general operators $A, B$ without any contractivity assumptions. 
Corollary 4. Let $A, B \in \mathscr{B}(\mathscr{H})^{+}$such that $T=\left[\begin{array}{cc}A & C^{*} \\ C & B\end{array}\right]$ is positive. Then

$$
\begin{aligned}
& w^{2 p}(C) \\
& \leq\left[\left\|A^{p}\right\|-\ell\left(\left\||(A-\|A\|)|^{p}\right\|\right)\right] \times\left[\left\|B^{p}\right\|-\ell\left(\left\||(B-\|B\|)|^{p}\right\|\right)\right]
\end{aligned}
$$

for all $p \geq 2$. In particular, we have

$$
w^{2 p}(C) \leq\left[\left\|A^{p}\right\|-\ell\left(\left\||(A-\|A\|)|^{p}\right\|\right)\right] .
$$

Moreover, in special case for $C=A$, we have

$$
w^{2 p}(A) \leq\left[\left\|A^{p}\right\|-\ell\left(\left.\|\|(A-\|A\|)\right|^{p} \|\right)\right] .
$$

Proof. The proof follows from Lemma 5 and Theorem 4.

Corollary 5. Let $T \in \mathscr{B}(\mathscr{H})$ be any operator. Then

$$
\begin{aligned}
w^{2 p}(T) \leq\left[\left\|T^{p}\right\|-\ell(\| \mid(T-\right. & \left.\left.\|T\|)\left.\right|^{p} \|\right)\right] \\
& \times\left[\left\|\left(T^{*}\right)^{p}\right\|-\ell\left(\left\|\left|\left(T^{*}-\|T\|\right)\right|^{p}\right\|\right)\right]
\end{aligned}
$$

for all $p \geq 2$.

Proof. Since $\left[\begin{array}{cc}|T| & T^{*} \\ T & \left|T^{*}\right|\end{array}\right] \in \mathscr{B}(\mathscr{H} \oplus \mathscr{H})$ is positive (see[24]), then the result follows from Corollary 4.

\section{Refinements of Numerical Radius inequalities FOR $n \times n$} MATRIX OPERATORS

Several numerical radius type inequalities improving and refining the inequality (1.1) have been recently obtained by many other authors see for example [2], [9], [10], [21]-[23], [28]. Among others, three important facts concerning the Numerical radius inequalities of $n \times n$ Operator matrices are obtained by different authors which are grouped together, as follows:

Let $A=\left[A_{i j}\right] \in \mathscr{B}\left(\bigoplus_{i=1}^{n} \mathscr{H}_{i}\right)$ such that $A_{i j} \in \mathscr{B}\left(\mathscr{H}_{j}, \mathscr{H}_{i}\right)$. Then

$$
w(A) \leq \begin{cases}\omega\left(\left[t_{i j}^{(1)}\right]\right), & \text { Hou \& Du in [18] } \\ \omega\left(\left[t_{i j}^{(2)}\right]\right), & \text { BaniDomi \& Kittaneh in }[5] ; \\ \omega\left(\left[t_{i j}^{(3)}\right]\right), & \text { AbuOmar \& Kittaneh in }[1]\end{cases}
$$


where

$$
t_{i j}^{(1)}=\omega\left(\left[\left\|T_{i j}\right\|\right]\right), \quad t_{i j}^{(2)}=\left\{\begin{array}{ll}
\frac{1}{2}\left(\left\|T_{i i}\right\|+\left\|T_{i i}^{2}\right\|^{1 / 2}\right), & i=j \\
\left\|T_{i j}\right\|, & i \neq j
\end{array},\right.
$$

and

$$
t_{i j}^{(3)}=\left\{\begin{array}{ll}
\omega\left(T_{i i}\right), & i=j \\
\left\|T_{i j}\right\|, & i \neq j
\end{array} .\right.
$$

In the next result we refine the latest bound $t_{i j}^{(3)}$ by adding a third part; which is the numerical range of the sub-operators on the opposite diagonal.

Theorem 5. Let $A=\left[A_{i j}\right] \in \mathscr{B}\left(\bigoplus_{i=1}^{n} \mathscr{H}_{i}\right)$ such that $A_{i j} \in \mathscr{B}\left(\mathscr{H}_{j}, \mathscr{H}_{i}\right)$, and $f, g$ be as in Lemma 5. Then

$$
w(A) \leq w\left(\left[a_{i j}\right]\right),
$$

where

$$
a_{i j}=\left\{\begin{array}{ll}
w\left(A_{i j}\right), & j=i \text { and } j \neq n-i+1 \\
w\left(A_{i j}\right), & j=n-i+1 \text { and } j \neq i \\
\left\|A_{i j}\right\|, & j \neq n-i+1 \text { and } j \neq i
\end{array} .\right.
$$

Proof. Let $x=\left[\begin{array}{llll}x_{1} & x_{2} & \cdots & x_{n}\end{array}\right]^{T} \in \bigoplus_{i=1}^{n} \mathscr{H}_{i}$ with $\|x\|=1$. For simplicity setting $k_{i}=n-i+1$, then we have

$$
\begin{aligned}
|\langle A x, x\rangle| & =\left|\sum_{i, j=1}^{n}\left\langle A_{i j} x_{j}, x_{i}\right\rangle\right| \\
& \leq \sum_{i, j=1}^{n}\left|\left\langle A_{i j} x_{j}, x_{i}\right\rangle\right| \\
& \leq \sum_{i=1}^{n}\left|\left\langle A_{i i} x_{i}, x_{i}\right\rangle\right|+\sum_{i=1}^{n}\left|\left\langle A_{k_{i} k_{i}} x_{k_{i}}, x_{k_{i}}\right\rangle\right|+\sum_{j \neq i, k_{i}}^{n}\left|\left\langle A_{i j} x_{j}, x_{i}\right\rangle\right| \\
& \leq \sum_{i=1}^{n} \omega\left(A_{i i}\right)\left\|x_{i}\right\|^{2}+\sum_{i=1}^{n} \omega\left(A_{k_{i} k_{i}}\right)\left\|x_{k_{i}}\right\|^{2}+\sum_{j \neq i}^{n}\left\|A_{i j}\right\|\left\|x_{i}\right\|\left\|x_{j}\right\| \\
& \leq \sum_{i=1}^{n} a_{i j}\left\|x_{i}\right\|\left\|x_{j}\right\| \\
& =\left\langle\left[a_{i j}\right] y, y\right\rangle
\end{aligned}
$$

where $y=\left(\begin{array}{llll}\left\|x_{1}\right\| & \left\|x_{2}\right\| & \cdots & \left\|x_{n}\right\|\end{array}\right)^{T}$. Taking the supremum over $x \in \bigoplus \mathscr{H}_{i}$, we obtain the desired result. 
Corollary 6. If $\mathbf{A}=\left[\begin{array}{cc}A_{11} & A_{12} \\ A_{21} & A_{22}\end{array}\right]$ in $\mathscr{B}\left(\mathscr{H}_{1}+\mathscr{H}_{2}\right)$, then

$$
\begin{aligned}
w\left(\left[\begin{array}{ll}
A_{11} & A_{12} \\
A_{21} & A_{22}
\end{array}\right]\right) \leq \frac{1}{2} & \left(w\left(A_{11}\right)+w\left(A_{22}\right)\right. \\
& \left.+\sqrt{\left(w\left(A_{11}\right)-w\left(A_{22}\right)\right)^{2}+\left(w\left(A_{12}\right)+w\left(A_{21}\right)\right)^{2}}\right)
\end{aligned}
$$

Proof. From Theorem 5, we have

$$
\begin{aligned}
w\left(\left[\begin{array}{ll}
A_{11} & A_{12} \\
A_{21} & A_{22}
\end{array}\right]\right) \leq & w\left(\left[\begin{array}{cc}
w\left(A_{11}\right) & w\left(A_{12}\right) \\
w\left(A_{21}\right) & w\left(A_{22}\right)
\end{array}\right]\right) \\
= & \frac{1}{2} r\left(\left[\begin{array}{cc}
w\left(A_{11}\right) & w\left(A_{12}\right)+w\left(A_{21}\right) \\
w\left(A_{21}\right)+w\left(A_{12}\right) & w\left(A_{22}\right)
\end{array}\right]\right) \\
= & \frac{1}{2}\left(w\left(A_{11}\right)+w\left(A_{22}\right)\right. \\
& \left.+\sqrt{\left(w\left(A_{11}\right)-w\left(A_{22}\right)\right)^{2}+\left(w\left(A_{12}\right)+w\left(A_{21}\right)\right)^{2}}\right)
\end{aligned}
$$

which proves the result.

Using the fact that for any $n \times n$ matrix $A=\left[A_{i j}\right]$ such that $A_{i j} \geq 0$. Then $w(A) \leq r\left(\frac{\left[A_{i j}\right]+\left[A_{j i}\right]}{2}\right)$, we may state Theorem 5 as follows:

Corollary 7. Let $A=\left[A_{i j}\right] \in \mathscr{B}\left(\bigoplus_{i=1}^{n} \mathscr{H}_{i}\right)$ and $f, g$ be as in Lemma 5. Then

$$
w(A) \leq r\left(\left[b_{i j}\right]\right)
$$

where

$$
b_{i j}= \begin{cases}w\left(A_{i j}\right), & j=i \text { and } j \neq n-i+1 \\ \frac{1}{2}\left(w\left(A_{i j}\right)+w\left(A_{j i}\right)\right), & j=n-i+1 \text { and } j \neq i \\ \frac{1}{2}\left(\left\|A_{i j}\right\|+\left\|A_{j i}\right\|\right), & j \neq i, n-i+1\end{cases}
$$

Theorem 6. Let $A=\left[A_{i j}\right] \in \mathscr{B}\left(\bigoplus_{i=1}^{n} \mathscr{H}_{i}\right)$ and $f, g$ be as in Lemma 5. Then

$$
w(A) \leq w\left(\left[c_{i j}\right]\right)
$$


NUMERICAL RADIUS INEQUALITIES FOR HILBERT SPACE OPERATORS 15

where

$$
c_{i j}=\left\{\begin{array}{ll}
\frac{1}{2}\left\|f^{2}\left(\left|A_{i i}\right|\right)+g^{2}\left(\left|A_{i i}^{*}\right|\right)\right\|, & j=i \quad \text { and } j \neq n-i+1 \\
\frac{1}{2}\left\|f^{2}\left(\left|A_{i j}\right|\right)+g^{2}\left(\left|A_{i j}^{*}\right|\right)\right\|, & j=n-i+1 \text { and } j \neq i \\
\left\|A_{i j}\right\|, & j \neq i, n-i+1
\end{array} .\right.
$$

Proof. Let $x=\left[\begin{array}{llll}x_{1} & x_{2} & \cdots & x_{n}\end{array}\right]^{T} \in \bigoplus_{i=1}^{n} \mathscr{H}_{i}$ with $\|x\|=1$. For simplicity setting $k_{i}=n-i+1$, then we have

$$
\begin{aligned}
& |\langle A x, x\rangle|=\left|\sum_{i, j=1}^{n}\left\langle A_{i j} x_{j}, x_{i}\right\rangle\right| \\
& \leq \sum_{i, j=1}^{n}\left|\left\langle A_{i j} x_{j}, x_{i}\right\rangle\right| \\
& \leq \sum_{i=1}^{n}\left|\left\langle A_{i i} x_{i}, x_{i}\right\rangle\right|+\sum_{i=1}^{n}\left|\left\langle A_{k_{i} k_{i}} x_{k_{i}}, x_{k_{i}}\right\rangle\right|+\sum_{j \neq i}^{n}\left|\left\langle A_{i j} x_{j}, x_{i}\right\rangle\right| \\
& \leq \sum_{i=1}^{n}\left\langle f^{2}\left(\left|A_{i i}\right|\right) x_{i}, x_{i}\right\rangle^{1 / 2}\left\langle g^{2}\left(\left|A_{i i}^{*}\right|\right) x_{i}, x_{i}\right\rangle^{1 / 2} \\
& +\sum_{i=1}^{n}\left\langle f^{2}\left(\left|A_{k_{i} k_{i}}\right|\right) x_{k_{i}}, x_{k_{i}}\right\rangle^{1 / 2}\left\langle g^{2}\left(\left|A_{k_{i} k_{i}}^{*}\right|\right) x_{k_{i}}, x_{k_{i}}\right\rangle^{1 / 2} \\
& +\sum_{j \neq i}^{n}\left|\left\langle A_{i j} x_{j}, x_{i}\right\rangle\right| \\
& \leq \frac{1}{2}\left[\sum_{i=1}^{n}\left\langle f^{2}\left(\left|A_{i i}\right|\right) x_{i}, x_{i}\right\rangle+\left\langle g^{2}\left(\left|A_{i i}^{*}\right|\right) x_{i}, x_{i}\right\rangle\right] \\
& +\frac{1}{2}\left[\sum_{i=1}^{n}\left\langle f^{2}\left(\left|A_{k_{i} k_{i}}\right|\right) x_{k_{i}}, x_{k_{i}}\right\rangle+\left\langle g^{2}\left(\left|A_{k_{i} k_{i}}^{*}\right|\right) x_{k_{i}}, x_{k_{i}}\right\rangle\right] \\
& +\sum_{j \neq i}^{n}\left|\left\langle A_{i j} x_{j}, x_{i}\right\rangle\right|
\end{aligned}
$$




$$
\begin{aligned}
& \leq \frac{1}{2} \sum_{i=1}^{n}\left\langle\left[f^{2}\left(\left|A_{i i}\right|\right)+g^{2}\left(\left|A_{i i}^{*}\right|\right)\right] x_{i}, x_{i}\right\rangle \\
& \quad+\frac{1}{2} \sum_{i=1}^{n}\left\langle\left[f^{2}\left(\left|A_{k_{i} k_{i}}\right|\right)+g^{2}\left(\left|A_{k_{i} k_{i}}^{*}\right|\right)\right] x_{k_{i}}, x_{k_{i}}\right\rangle \\
& \quad+\sum_{j \neq i}^{n}\left|\left\langle A_{i j} x_{j}, x_{i}\right\rangle\right| \\
& =\left\langle\left[c_{i j}\right] y, y\right\rangle
\end{aligned}
$$

where $y=\left(\begin{array}{llll}\left\|x_{1}\right\| & \left\|x_{2}\right\| & \cdots & \left\|x_{n}\right\|\end{array}\right)^{T}$. Taking the supremum over $x \in \bigoplus \mathscr{H}_{i}$, we obtain the desired result.

As we did in Corollary 7 we may restate Theorem 6 in terms of spectral radius as follows:

Corollary 8. Let $A=\left[A_{i j}\right] \in \mathscr{B}\left(\bigoplus_{i=1}^{n} \mathscr{H}_{i}\right)$ and $f, g$ be as in Lemma 5. Then

$$
w(A) \leq r\left(\left[d_{i j}\right]\right)
$$

where

$$
d_{i j}= \begin{cases}\frac{1}{2}\left\|f^{2}\left(\left|A_{i j}\right|\right)+g^{2}\left(\left|A_{i j}^{*}\right|\right)\right\|, & j=i \text { and } j \neq n-i+1 \\ \frac{1}{4}\left[\left\|f^{2}\left(\left|A_{i j}\right|\right)+g^{2}\left(\left|A_{i j}^{*}\right|\right)\right\|\right. & \\ \left.\quad+\left\|f^{2}\left(\left|A_{j i}\right|\right)+g^{2}\left(\left|A_{j i}^{*}\right|\right)\right\|\right], & j=n-i+1 \text { and } j \neq i . \\ \frac{1}{2}\left(\left\|A_{i j}\right\|+\left\|A_{j i}\right\|\right), & j \neq i, n-i+1\end{cases}
$$

Acknowledgment: The author wish to thank the referee for his fruitful comments and careful reading of the original manuscript of this work that have implemented the final version of this work.

\section{REFERENCES}

[1] A. Abu-Omar and F. Kittaneh, Numerical radius for $n \times n$ operator matrices, Linear Algebra Appl., 468 (2015), 18-26.

[2] A. Abu-Omar and F. Kittaneh, Estimates for the numerical radius and the spectral radius of the Frobenius companion matrix and bounds for the zeros of polynomials, Ann. Funct. Anal., 5 (2014), 56-62.

[3] M.W. Alomari, Numerical radius inequalities for Hilbert space operators, Preprint (2018), arXiv:1810.05710.

[4] R. Bhatia, Matrix Analysis, Springer, New York, 1997. 
[5] W. Bani-Domi and F. Kittaneh, Numerical radius inequalities for operator matrices, Linear's Multilinear Algebra, 57 (2009), 421-427.

[6] M.-T. Chien, H.-L. Gau, C.-K. Li, M.-C. Tsai and K.-Z. Wang, Product of operators and numerical range, Linear\& Multilinear Algebra, 64 (1) (2016), $58-67$.

[7] M.-T. Chien, C.-L. Ko, H. Nakazato, On the numerical ranges of matrix products, Appl. Math. Lett., 23 (2010), 732-737.

[8] S.S. Dragomir, Inequalities for the Numerical Radius of Linear Operators in Hilbert Spaces, SpringerBriefs in Mathematics, 2013.

[9] S.S. Dragomir, Some inequalities for the Euclidean operator radius of two operators in Hilbert spaces, Linear Algebra Appl., 419 (2006), 256-264.

[10] S.S. Dragomir, Power inequalities for the numerical radius of a product of two operators in Hilbert spaces, Sarajevo J. Math., 5 (18)(2) (2009), 269-278.

[11] S.S. Dragomir, Some inequalities for the norm and the numerical radius of linear operator in Hilbert spaces, Tamkang J. Math.,39 (1) (2008), 1-7.

[12] S.S. Dragomir, Some refinements of schwarz inequality, Simposional de Math Si Appl. Polytechnical Inst Timisoara, Romania, 12 (1985), 13-16.

[13] M. El-Haddad and F. Kittaneh, Numerical radius inequalities for Hilbert space operators. II. Studia Math., 182 (2) (2007), 133-140.

[14] K.E. Gustafson D.K. Rao, Numerical Range, Springer-Verlag, New York, 1996.

[15] O. Hirzallah, F. Kittaneh and K. Shebrawi, Numerical radius inequalities for certain $2 \times 2$ operator matrices, Integral Equations Operator Theory 71 (2011), $129-149$.

[16] R.A. Horn and C.R. Johnson, Matrix Analysis, Cambridge University Press, Cambridge, 1985.

[17] R.A. Horn and C.R. Johnson, Topics in Matrix Analysis, Cambridge University Press, Cambridge, 1991.

[18] J.C. Hou and H.K. Du, Norm inequalities of positive operator matrices, Integral Equations Operator Theory, 22 (1995), 281-294.

[19] F. Kittaneh, Numerical radius inequalities for Hilbert space operators, Studia Math., 168 (1) (2005), 73-80.

[20] F. Kittaneh, Spectral radius inequalities for Hilbert space operators, Proc. Amer. Math. Soc., 134 (2) (2005), 385-390.

[21] F. Kittaneh, A numerical radius inequality and an estimate for the numerical radius of the Frobenius companion matrix, Studia Math. 158 (2003), 11-17.

[22] F. Kittaneh, Bounds for the zeros of polynomials from matrix inequalities, Arch.Math., (Basel) 81 (2003), 601-608.

[23] F. Kittaneh, Norm inequalities for certian operator sums, J. Funct. Anal. 143 (2) (1997), 337-348.

[24] F. Kittaneh, Notes on some inequalities for Hilbert Space operators, Publ. Res. Inst. Math. Sci, 24 (2) (1988), 283-293.

[25] T. Kato, Notes on some inequalities for linear operators, Math. Ann., 125 (1952), 208-212. 
[26] M.S. Moslehian, M. Sattari and K. Shebrawi, Extensions of Euclidean operator radius inequalities, Mathematica Scandinavica, 120 (1) (2017), 129-144.

[27] C.-K. Li, M.-C. Tsai , K.-Z. Wang, N.-C. Wong, The spectrum of the product of operators, and the product of their numerical ranges, Linear Algebra Appl., 469 (2015), 487-499.

[28] M.E. Omidvar, M.S. Moslehian and A. Niknam, Some numerical radius inequalities for Hilbert space operators, Involve, 2 (4) (2009), 471-478.

[29] M. Sattari, M.S. Moslehian and T. Yamazaki, Some genaralized numerical radius inequalities for Hilbert space operators, Linear Algebra Appl, 470 (2014), $1-12$.

[30] T. Yamazaki, On upper and lower bounds of the numerical radius and an equality condition, Studia Math., 178 (2007), 83-89.

Department of Mathematics, Faculty of Science and Information Technology, Irbid National University, 2600 Irbid 21110, Jordan.

E-mail address: mwomath@gmail.com 